\title{
Acquis en électrocinétique à courant continu
}

\section{B. Calmettes}

\section{(2) OpenEdition}

Journals

Édition électronique

URL : http://journals.openedition.org/trema/2323

DOI : $10.4000 /$ trema.2323

ISSN : 2107-0997

\section{Éditeur}

Faculté d'Éducation de l'université de Montpellier

\section{Édition imprimée}

Date de publication : 1 mai 1993

Pagination : 37-48

ISSN : 1167-315X

\section{Référence électronique}

B. Calmettes, « Acquis en électrocinétique à courant continu », Tréma [En ligne], 3-4 | 1993, mis en ligne le 01 mai 1993, consulté le 20 avril 2019. URL : http://journals.openedition.org/trema/2323 ; DOI :

10.4000/trema.2323

Ce document a été généré automatiquement le 20 avril 2019

Trema 


\section{Acquis en électrocinétique à courant continu}

\section{B. Calmettes}

1 Les élèves des classes de 1ère F3 des Lycées Techniques (Cycle préparant au Bac Technologique, option Electrotechnique) sont issus de deux groupes. Les élèves de $1 \mathrm{~F} 3 \mathrm{~N}$ ont suivi des enseignements dans une classe de seconde TSA (seconde classique pour l'enseignement de la physique et des enseignements optionnels de type technologique) tandis que les élèves de $1 \mathrm{~F} 3 \mathrm{P}$ ( $1^{\text {ère }}$ d'adaptation) ont transité par l'enseignement professionnel et possèdent un BEP (électrotechnique généralement). Tous les élèves suivent en classe de première un enseignement de contenu commun en Physique Appliquée, l'objectif étant de leur fournir les bases qui leur permettront en Terminale d'étudier des systèmes électroniques et électrotechniques évolués (hacheur, onduleur, machines électriques,...).

\section{Approche générale}

\section{Les passés scolaires différents des élèves de 1F3P et de 1F3N marquent-ils leurs connaissances en physique appliquée?}

2 Afin de préciser ce point, nous avons mené une série d'entretiens avec des professeurs et des responsables de différentes classes (BEP, 1ère et $\mathrm{TF} 3$ ), de différentes matières (Physique Appliquée, Automatisme et Informatique, Mathématiques).

3 Les informations recueillies peuvent être résumées de la façon suivante :

- Les élèves des sections P issus de BEP sont motivés pour les Travaux Pratiques et y possèdent de solides connaissances. Celles-ci peuvent servir de base à tout apport de cours postérieur, y compris théorique, à condition qu'ils en ressentent une nécessité (pouvoir explicatif et prévisionnel de modèles par exemple) sinon, ils se contenteraient d'un descriptif sommaire, voire superficiel, tant leurs difficultés expressionnelles et d'abstraction leur posent souvent de sérieux problèmes.

- Ces élèves éprouveraient aussi des difficultés dans les formulations mathématiques, les résolutions d'équations surtout si la liaison avec leurs acquis de pratique en physique n'est pas immédiate. Ce 
manque de maîtrise au niveau de l'abstraction et les lacunes d'ordre mathématique seraient à la source d'un manque de méthodologie scientifique, d'un «cherche-formule » effréné et d'une vision théorique limitée.

- les élèves de 1F3N réussissent mieux dans les approches théoriques et mathématiques de la physique. Leurs qualités en ce point neutralisent un désir de recherche par la pratique. Les problèmes devraient, d'après eux, pouvoir se "résoudre uniquement sur le papier».

De ces entretiens relatifs à la pratique journalière de classe, il ressort que les élèves de $1 \mathrm{~F} 3 \mathrm{~N}$ et $1 \mathrm{~F} 3 \mathrm{P}$ présenteraient des attitudes, des connaissances et des aptitudes différentes.

\section{Problématique de la recherche}

\section{Il existerait des différences entre les connaissances et les approches théoriques des élèves des classes de $1 \mathrm{~F} 3 \mathrm{~N}$ et de $1 \mathrm{~F} 3 \mathrm{P}$. Peut-on les mettre en évidence par un questionnaire papier/crayon ?}

5 Afin de tenter de préciser certaines de ces connaissances, nous avons construit, à partir de questions déjà posées par d'autres chercheurs et d'items originaux, un questionnaire devant permettre après enseignement, sur la partie de leur programme relative à l'électrocinétique à courant continu, de comparer les deux groupes d'élèves (Calmettes, 1992).

\section{L'outil}

6 Le questionnaire auquel ont répondu environ 80 élèves (3 classes de 3 Lycées Techniques de l'Académie de Toulouse) de chaque groupe comportait 20 questions. Les élèves avaient la possibilité de ne pas répondre aux questions s'ils ne pensaient pas avoir la bonne solution. Il abordait les sujets suivants : notions de tension, de courant et de résistor; approches des raisonnements adoptés dans différentes situations; schématisation; approches de l'abstraction par l'algébrisation du courant, de la tension et par la loi d'Ohm. Des entretiens complémentaires post-test ont permis de préciser certains comportements.

7 Le questionnaire a été posé à la fin du premier trimestre de l'année scolaire 91/92 après enseignement de la partie électrocinétique en courant continu.

8 Les questions que nous aborderons ici sont choisies parmi celles montrant le plus les différences entre les deux groupes d'élèves ou celles présentant un intérêt particulier pour la recherche.

\section{Etude de quelques résultats}

Les nombres dans les tableaux correspondent à des pourcentages. Dans les circuits proposés, les générateurs et les appareils de mesure sont considérés comme étant parfaits. 


\section{Raisonnement sur un circuit série}

10 La question posée (proposée par Closset, J. L., 1983) a pour objet la détection du mode de raisonnement adopté par les élèves face à un circuit série.

11 Les raisonnements conduisant à des réponses inexactes - effectivement observés - sont le raisonnement " séquentiel » ou « à épuisement de courant ", le raisonnement « à courant constant » (décrits dans de précédents travaux, ceux de Jean-Louis Closset, Jean-Jacques Dupin et Samuel Joshua, Laurence Viennot par exemple).

Nous appellerons « raisonnement globaliste » celui menant à une réponse exacte obtenue par une description systémique du circuit et « raisonnement formel » un raisonnement aboutissant à une réponse exacte mais consistant en un traitement mathématique de toutes les équations que l'on peut écrire concernant le circuit. Si ce raisonnement formel amène au bon résultat, on peut légitimement penser que l'écriture de chacune des relations a été correctement écrite à travers une interprétation physique elle-même juste. Mais, on ne peut rien dire sur la connaissance physique globale du circuit proposé. Il est possible que la procédure de résolution algébrique cache les difficultés cognitives et la connaissance de la conception de l'élève sur le sujet proposé.

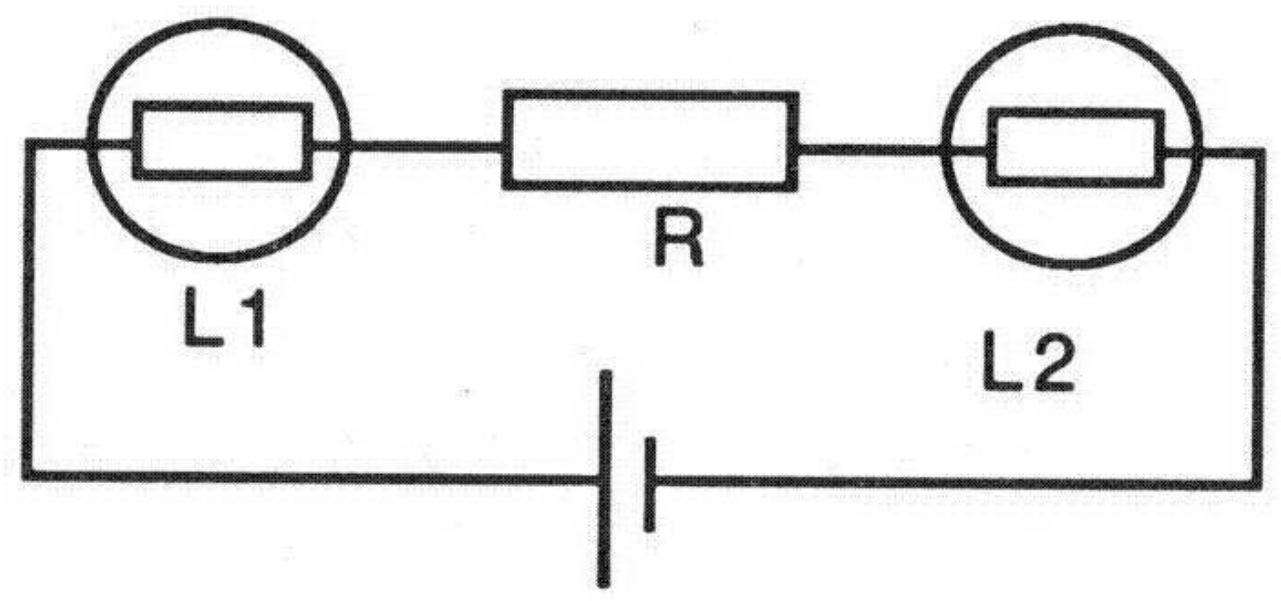

a- L1 brille-t-elle plus fort, aussi fort, moins fort que $L 2$

On augmente $R$

b - L1 brille-t-elle plus fort, aussi fort, moins fort qu'avant?

c - L2 brille-t-elle plus fort, aussi fort, moins fort qu'avant?

$R$ est un résistor, $L 1$ et $L 2$ des ampoules électriques identiques

\section{Réponses :}

La question a été également posée (travaux de Jean-Louis Closset) à des populations d'une quarantaine d'élèves de fin d'enseignement secondaire en Belgique et à des étudiants de D.E.U.G. S.S.M. (ce D.E.U.G. correspond à une seconde année de premier cycle universitaire en sciences exactes avec un cours à option d'électronique).

14 Le tableau suivant permet de situer nos résultats par rapport à ceux de ces deux populations.

$151 \mathrm{~F} 3 \mathrm{~N}=1$ ère $\mathrm{F} 3$ après seconde T.S.A.

1F3P = 1ère F3 d'adaptation (après B.E.P.) 
fin ens. sec = fin d'enseignement secondaire en Belgique A2élec = seconde année universitaire, option électronique en France

\begin{tabular}{|l|c|c|c|c|}
\cline { 2 - 5 } \multicolumn{1}{c|}{} & fin ens.sec & 1F3N & 1F3P & A2élec \\
\hline Réponse exacte & $\mathbf{1 7}$ & $\mathbf{1 7}$ & $\mathbf{5 8}$ & $\mathbf{7 5}$ \\
Séquentiel & 51 & 49 & 12 & 11 \\
Courant constant & 15 & 6 & 6 & 7 \\
\hline
\end{tabular}

Les résultats (sur les parties $\mathrm{a}, \mathrm{b}$ et $\mathrm{c}$ ) sont présentés dans un ordre permettant d'apprécier la progression en terme de « réponse exacte».

Les principales réponses fournies peuvent être analysées de la façon suivante :

- «L1 brille autant que L2. » * «L1 et L2 brillent moins. » Il s'agit de la réponse exacte.

- «L1 brille plus fort que L2. » * «L1 brille autant et L2 moins, » Il s'agit du raisonnement à usure de courant. Celui-ci doit pour atteindre la lampe 2 franchir une résistance et il y perd une partie de son énergie. L'intensité lumineuse de la lampe 2 est alors plus faible que celle de la lampe 1. Ce raisonnement s'accompagne de la réponse : «L1 brille autant et L2 moins » quand on augmente la valeur de la résistance $\mathrm{R}$. Ce type de raisonnement séquentiel est extrêmement répandu dans les classes de $1 \mathrm{~F} 3 \mathrm{~N}$ et beaucoup moins dans les classes de 1F3P.

- « L1 brille autant que L2. » * «L1 et L2 autant qu'avant. » Il s'agit d'un raisonnement à courant constant. La pile apparait comme un générateur de courant débitant un courant constant quelle que soit sa charge.

\section{Commentaires :}

8 On pourra observer que les sommes des réponses présentées sont de $72 \%$ pour les $1 \mathrm{~F} 3 \mathrm{~N}$ et de $76 \%$ pour les 1F3P. Le complément est à chercher dans des changements de raisonnements ou une mauvaise compréhension de la question lors du passage de la partie a aux parties b et $\mathrm{c}(12 \%$ des $1 \mathrm{~F} 3 \mathrm{P}$ et $5 \%$ des $1 \mathrm{~F} 3 \mathrm{~N}$ semblent par exemple produire un raisonnement correct à la partie a et passent à un raisonnement séquentiel pour les parties $b$ et $c$ ) et dans des réponses ne correspondant pas directement à la classification proposée. Certains élèves en particulier semblent raisonner séquentiellement à partir du sens de déplacement des électrons (mis dans la rubrique séquentiel par Jean-Louis Closset), raisonnement «à épuisement d'électrons» (environ $5 \%$ dans chacun des groupes).

9 Les raisonnements à courant constant sont relativement peu produits au niveau des classes de 1F3. Les raisonnements globalistes croissent en pourcentage au fur et à mesure que disparait le raisonnement séquentiel.

Il est intéressant de noter que les élèves issus des classes de B.E.P. ont acquis de façon prépondérante un raisonnement permettant d'arriver à la réponse exacte.

\section{Conventions et changements de signes}

21 La question choisie (d'après Viennot L., 1981) a été construite afin de comprendre comment les écritures algébriques étaient perçues par les élèves de différents niveaux.

Convention: On convient de déclarer l'intensité i du courant positive lorsque celuici va de A vers B. Pour une situation physique donnée, on a les 3 relations suivantes 
justes et compatibles

(1) $>0$

(2) $V A-V B=R . i$

(3) $\mathrm{VA}-\mathrm{VB}>0$

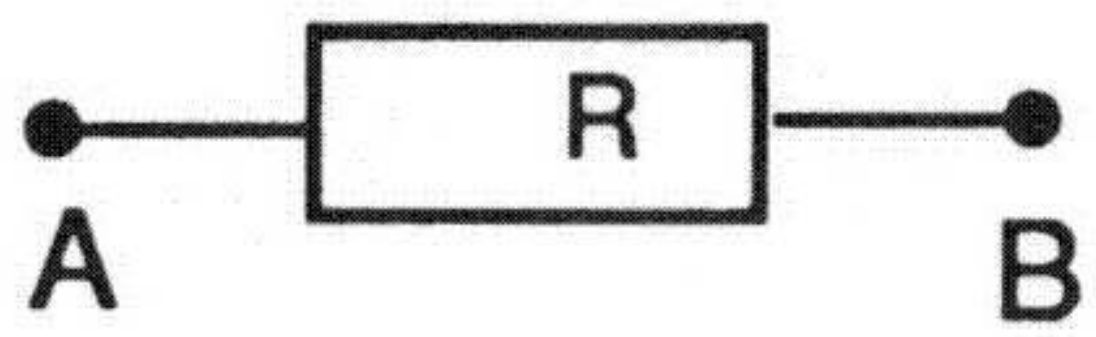

Qu'est-ce qui change dans chacune de ces relations, dans chacun des cas suivants a et $b$ à considérer séparément ?

Justifiez

Relation 1 Relation 2 Relation 3

a- On change le sens choisi conventionnellement pour les intensités tout en gardant la même situation physique (le courant réel va de $A$ vers $B$ )

b- On garde la même convention de signe que dans l'énoncé, on change le sens réel du courant qui va donc de B vers A.

Dans un texte reprenant le même thème (Saltiel E. et Viennot L., 1991), il est noté : « La valeur numérique d'une grandeur algébrique dépend de la situation physique elle-même et de précisions sur la définition de la grandeur, qu'on nomme souvent conventions de signe et qui reviennent en fait à expliquer complètement ce que désigne le symbole utilisé. »

Une intensité I ne décrit complètement la grandeur physique associée que si l'on s'est donné un sens positif. «Une fois les grandeurs algébriques et leurs symboles définis, les relations ne dépendent plus de la situation réelle particulière étudiée. »

La question posée correspond aux réponses exactes suivantes :

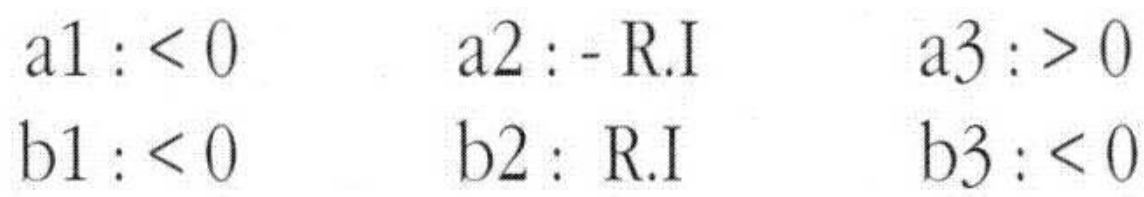

Dans le tableau de résultats suivant n'ont été indiqués que les pourcentages de réponses exactes. 


$\begin{array}{lllllll} & \text { a1 } & \text { a2 } & \text { a3 } & \text { b1 } & \text { b2 } & \text { b3 } \\ \text { 1F3N } & 43 & 35 & 25 & 29 & 25 & 38 \\ \text { 1F3P } & 32 & 15 & 17 & 15 & 17 & 26\end{array}$

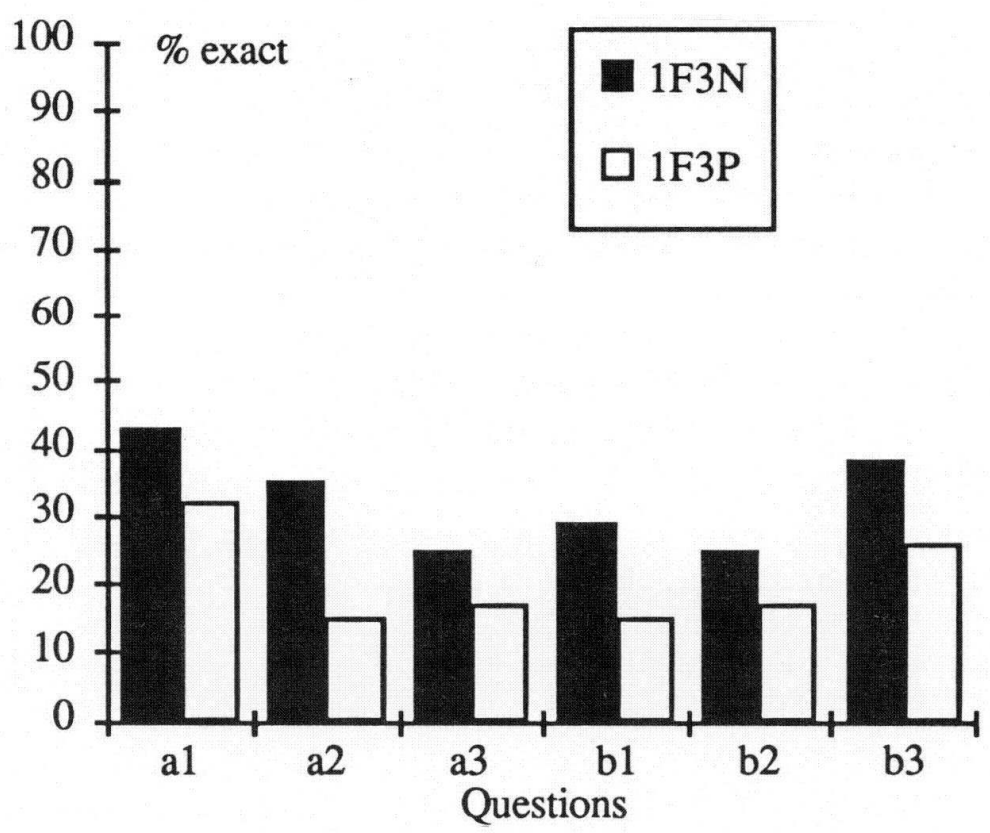

Commentaires :

Beaucoup d'élèves n'ont pas répondu à cette question, la trouvant difficile. Les réponses exactes sont donc relativement peu nombreuses. L'effet d'un apprentissage nouveau ne semble pas être seul responsable de cette situation; les résultats obtenus à partir du même item posé à des élèves de TF3 sont proches de ceux exposés ci-dessus. Les élèves de 1 F3N obtiennent à tous les items de meilleurs pourcentages de réponses exactes.

\section{Raisonnements sur les circuits et relations mathématiques}

La réponse exacte à la question (proposée par Viennot, L., 1983) :

peut être obtenue par un raisonnement formel faisant appel aux mathématiques en utilisant une différentielle (non possédé), par un raisonnement mathématique par "essais" de valeurs de R1 après expression de la tension U éventuellement simplifiée (jamais utilisé) ou par un raisonnement globaliste. 


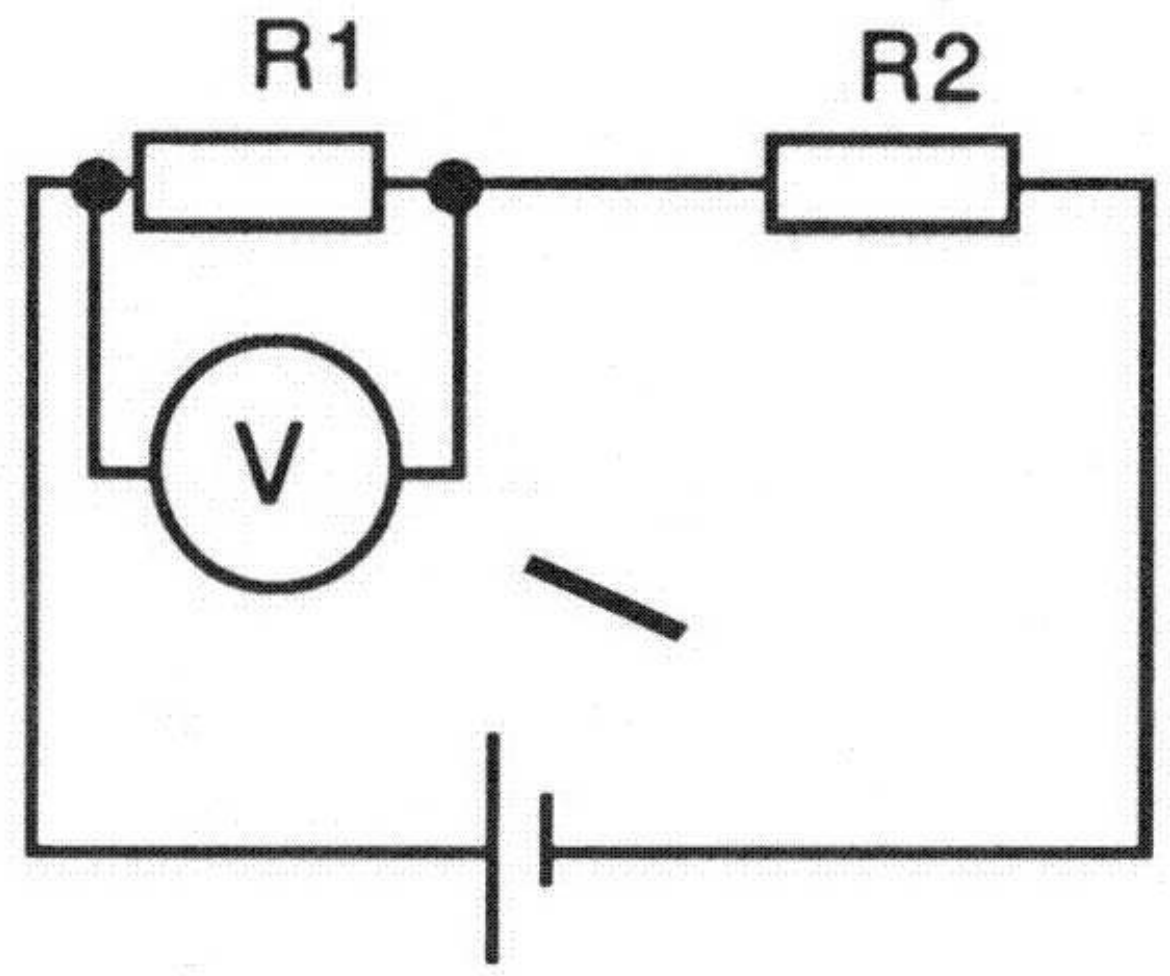

Exercice : On augmente R1.

On relève que la tension mesurée $U$ augmente aussi.

Expliquer pourquoi.

Réponse d'un élève

$U=R 1 . I$. Si R1 augmente, $U$ aussi.

Cette réponse vous paraît-elle correcte? Expliquez.

La seule alternative est alors de raisonner par étapes ; le raisonnement « correct » serait :

1. Quand R1 augmente, le courant dans le circuit diminue.

2. La tension aux bornes du résister $\mathrm{R} 2$ de résistance constante diminue alors.

3. La tension $\mathrm{E}$ étant constante, la tension aux bornes de R1 augmente.

Les résultats montrent que les élèves qui ont envisagé le point 2 envisagent le point 3.

\section{Réponses :}

Les résultats ont été classés de la manière suivante :

- Les élèves ayant fourni une réponse exacte avec un raisonnement correct (réponse exacte).

- Les élèves ayant fourni un raisonnement se terminant à l'étape 1 et aboutissant à une contradiction à laquelle ils ne pouvaient répondre : $s i$ I diminue et que R1 augmente, que fait le produit R1.I (1ère étape seulement)?

- Les élèves qui raisonnent franchement en courant constant et qui l'écrivent de façon plus ou moins explicite (raisonnement « en courant constant »).

- Les élèves qui sont satisfaits de la réponse proposée dans l'énoncé (oui sans explication). 
Réponse exacte

Première étape

Raisonnement "en courant constant"

Oui sans explication

Autre/JSP/Pas de réponse

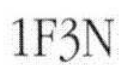

1F3P

1

5

5

28

61

40

18

21

15

\section{Commentaires :}

On peut observer que très peu d'élèves arrivent au bout de la solution complète. Les élèves de $1 \mathrm{~F} 3 \mathrm{P}$ sont plus nombreux à produire la réponse exacte et le raisonnement correspondant à l'étape 1. Le raisonnement « en courant constant » appelé aussi par JeanLouis Closset « raisonnement séquentiel de type 2 » est, cependant, prépondérant dans chaque groupe. La question posée a poussé des élèves qui, jusqu'alors, avaient utilisé à bon escient un raisonnement de type globaliste, et qui donc en particulier avaient noté que l'effet de l'augmentation de la résistance $\mathrm{R}$ était de faire diminuer l'intensité du courant, à raisonner différemment. En fait, les entretiens post-test que nous avons menés nous ont montré que c'est le formalisme, l'utilisation possible d'une relation qui a fait perdre majoritairement aux étudiants tout contact avec la réalité physique. Ils ne portent plus sur la question posée de raisonnement physique, le problème devient pour eux, uniquement mathématique. Leur logique est une logique de mise en œuvre immédiate et non une logique de réflexion visant à comparer toutes les données avec l'ensemble des connaissances dont ils disposent. On peut alors s'interroger d'une façon plus générale sur l'utilisation privilégiée de certains outils mathématiques au détriment des outils conceptuels de la physique, sur l'intégration dans les pratiques de l'enseignement des deux approches. La facilité apparente qu'apportent les premiers semble restreindre la disponibilité des seconds.

\section{Utilisations de relations mathématiques}

Donnez les relations permettant de calculer la résistance équivalente dans chacun des cas de groupements ci dessous
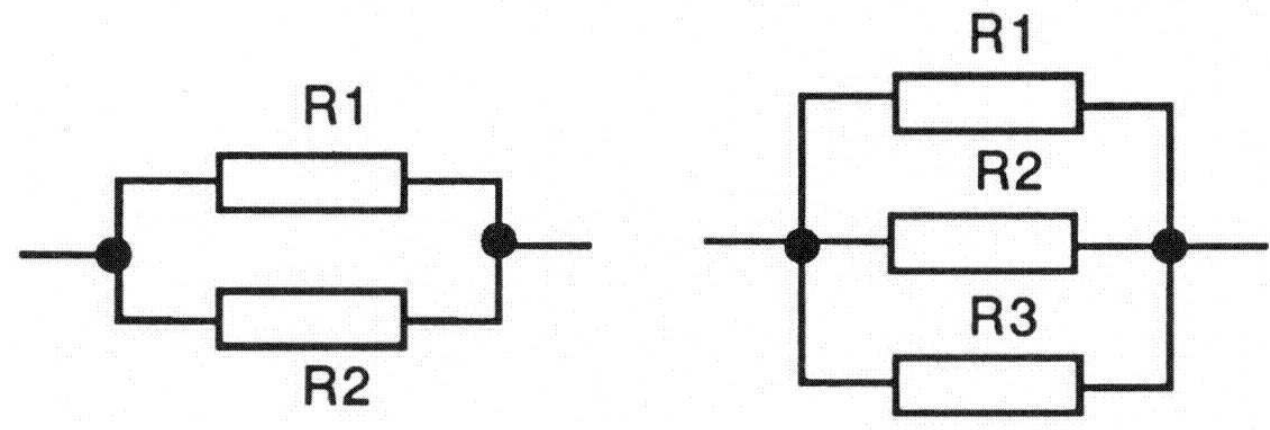
La question porte sur le formalisme utilisé dans les calculs de résistances équivalentes dans les associations de résistors. Elle doit permettre de connaître le choix des relations suivant les situations. Les relations principales relevées sont :

$$
\begin{aligned}
& \frac{1}{\mathrm{R}}=\frac{1}{\mathrm{R} 1}+\frac{1}{\mathrm{R} 2} \text { (1) } \quad \mathrm{R}=\frac{\mathrm{R} 1 \cdot \mathrm{R} 2}{\mathrm{R} 1+\mathrm{R} 2} \text { (2) } \\
& \frac{1}{R}=\frac{1}{R 1}+\frac{1}{R 2}+\frac{1}{R 3} \text { (3) } \\
& \mathrm{R}=\frac{\mathrm{R} 1 \cdot \mathrm{R} 2 \cdot \mathrm{R} 3}{\mathrm{R} 1+\mathrm{R} 2+\mathrm{R} 3} \text { (4) } \mathrm{R}=\frac{1}{\mathrm{R} 1}+\frac{1}{\mathrm{R} 2}+\frac{1}{\mathrm{R} 3} \text { (5) }
\end{aligned}
$$

Réponses :

Les résultats sont les suivants (les réponses exactes sont notées en caractères gras) :

2 R EN PARALLELE FORME (1)

$1 \mathrm{~F} 3 \mathrm{~N} \quad 1 \mathrm{~F} 3 \mathrm{P}$

2 R EN PARALLELE FORME (2)

$18 \quad 6$

3 R EN PARALLELE FORME (3)

$75 \quad 90$

$3 \mathrm{R}$ en parallèle forme (4)

$57 \quad 75$

$3 \mathrm{R}$ en parallèle forme (5)

Autres formes

18

10

8

14

Relations (2) et (3) présentes ensemble

39

69

Relations (1) et (3) associées

18

Relations (2) et (4) associées

18

\section{Commentaires :}

- La relation (2) donnant directement la valeur de R est utilisée de façon prépondérante, surtout chez les 1F3P.

- Des confusions apparaissent effectivement dans les écritures des relations pour 3 résistors en parallèle, surtout chez les $1 \mathrm{~F} 3 \mathrm{~N}$. L'utilisation quasi obligatoire de la relation avec des inverses mathématiques accroît le nombre de réponses inexactes.

- Majoritairement, deux relations sont assimilées : une pour 2 résistors en parallèle (relation (2)) et une pour 3 résistors en parallèle (relation (3)). La relation par les inverses est peu utilisée (surtout chez les 1F3P) pour 2 résistors en parallèle.

- Tous les élèves qui utilisent la relation (1) utilisent ensuite la relation (3). On relève ici des changements dans les types de relations mathématiques utilisées. La relation mathématique utilisée de façon prépondérante dans le cas de 2 résistors (forme (2)) permet, semble-t-il, de donner une réponse rapide ( $\mathrm{R}$ est donnée directement, donc coût de réflexion réduit) et d'éviter des étapes complémentaires, des calculs mathématiques peut-être risqués. 
Pour deux situations très proches, pratiquement semblables, ce sont donc deux types de relations qui sont mémorisés et utilisés.

\section{Conclusions}

\section{Comparaison des deux groupes d'élèves}

Sur les réponses aux questions présentées dans ce document, les 1F3P et les $1 \mathrm{~F} 3 \mathrm{~N}$ présentent des différences notables sur les points suivants :

- A propos de la question relative aux raisonnements (paragraphe 3.1), les 1F3P semblent posséder un raisonnement sur les circuits série de type globaliste $(60 \%)$ qui doit leur permettre de faire de meilleures prévisions sur l'évolution des systèmes électriques simples. Sur ce point, les $1 F 3 \mathrm{~N}$ raisonnent de façon prépondérante en utilisant le modèle séquentiel $(50 \%)$.

- Les $1 \mathrm{~F} 3 \mathrm{~N}$ ont de meilleures performances que les $1 \mathrm{~F} 3 \mathrm{P}$ sur les problèmes liés à l'abstraction : loi d'Ohm algébrisée (paragraphe 3.2).

- Les réponses aux questions relatives aux relations utilisées dans les associations de résistors (paragraphe 3.4) s'accompagnent d'un pourcentage de réponses exactes légèrement supérieur pour les $1 \mathrm{~F} 3 \mathrm{P}$.

\section{Influence de l'enseignement}

L'étude présentée semble montrer que le type d'enseignement antérieur reçu (au sens très large comprenant à la fois les contenus des programmes, la forme d'activité de transmission du savoir et la motivation des élèves) influence le comportement des étudiants quant à leurs types de raisonnements, sur les circuits en particulier.

Les élèves de 1F3P obtiennent généralement sur ces derniers items des pourcentages de réponses exactes élevés et toujours supérieurs à ceux obtenus par les élèves de $1 \mathrm{~F} 3 \mathrm{~N}$. Cette réussite ne semble pas pour autant liée à une meilleure maitrise de la notion de tension (mis à part le fait qu'ils savent ce qu'est un générateur de tension). En effet, la plupart des raisonnements que nous avons pu approfondir lors d'entretiens se font en courant mais avec une vision systémique du circuit. Les élèves conçoivent généralement que la modification d'un composant d'un circuit modifie les points de fonctionnement de tous les composants du circuit. Les raisonnements en tension sont le plus souvent formels, associés à la loi d'Ohm. Les limites du fonctionnement de leurs raisonnements sont liées à la présence de relations mathématiques ou/et à la nécessité de résolution d'un problème en plusieurs étapes.

41 Relevons toutefois que les bons raisonnements des 1F3P sur les circuits restent insuffisants pour leur permettre l'analyse formelle et plus symbolique des phénomènes physiques telle qu'elle est explorée précédemment.

\section{A propos des autres observations}

42 Nous avons mis en évidence dans les deux groupes avec des effets plus ou moins marqués des changements d'attitudes cognitives montrant une certaine sensibilité des choix 
(conscients ou inconscients) opérés par les élèves dans des situations mettant en cause les raisonnements sur les circuits et les relations mathématiques utilisées en physique.

Qu'est-ce qui paraît provoquer ces changements dans les situations proposées?

- Considérons le changement de raisonnement sur les circuits électriques entre les questions présentées aux paragraphes Raisonnement sur un circuit série et Raisonnements sur les circuits et relations mathématiques. Ce qui peut y être mis en cause, c'est le besoin chez les élèves de fournir un résultat rapide en rapport direct avec la question posée et utilisant la relation présentée. Le raisonnement en plusieurs étapes, plus construit, est peu produit car nécessitant un investissement plus lourd.

- Dans le cas du calcul de la résistance équivalente aux associations de résistors en parallèle, nous avons relevé que deux relations étaient généralement apprises. La relation mathématique qui fonctionne quel que soit le nombre de résistors (passant par l'inverse mathématique de R) est utilisée par un grand nombre d'élèves seulement si le nombre de résistors est supérieur à 2 . Nous avons remarqué à ce propos lors des entretiens une certaine gêne quant à l'utilisation mathématique des inverses. Ceux-ci étant mal maîtrisés, une forme en produit dans laquelle la résistance $\mathrm{R}$ est donnée directement est utilisée pour l'association à 2 résistors. Nous aurions ici en fait un besoin de la part des élèves de fournir un résultat rapide et direct (pas de passage par l'inverse si possible) sans investissement en mécanisme opératoire, quitte à élever le coût de mémorisation des relations mathématiques

On peut faire l'hypothèse que ces instabilités interviennent au niveau du traitement $d u$ problème posé à l'individu. Ce traitement est dirigé par les données du problème, la question posée, la référence mémorisée, les concepts a priori mobilisables par l'élève. La mise en relation de ces divers éléments devrait produire la réponse exacte. L'étude des différents résultats obtenus aux items proposés semble bien indiquer que les difficultés pourraient être liées à la fois à la perte du contact avec le réel, au besoin d'utiliser des relations mathématiques et à l'augmentation du nombre d'opérations mentales à réaliser.

45 A propos de "coût cognitif ", il convient de séparer l'investissement en terme de mémorisation souvent accepté et le coût de production d'une solution réfléchie, en plusieurs étapes, souvent refusé.

\section{Pespectives}

Les résultats de cette étude peuvent ouvrir des recherches dans différentes directions :

- le cursus des élèves passés par l'enseignement professionnel court et ayant rejoint l'enseignement technologique pourrait être évalué à différentes étapes afin de préciser les influences éventuelles des types d'apprentissage (travaux pratiques, séances en atelier, cours,...);

- la pratique industrielle paraît provoquer au niveau de l'enseignement de la physique appliquée une série de transpositions didactiques : savoir industriel -savoir institué - savoir enseigné - savoir appris par les élèves. Ces évolutions et variations de savoirs peuvent également faire l'objet d'une étude.

De façon plus générale, notre travail peut s'insérer dans l'ensemble de la recherche sur les modes de raisonnements, les conceptions des élèves et les influences des pratiques sociales sur ceux-ci. 


\section{BIBLIOGRAPHIE}

\section{Références}

CALMETTES B. : Acquis en électrocinétique à courant continu : comparaison 1ère F3 / 1ère d'adaptation F3. Mémoire de D.E.A. (Dir. B. Bouldoires). Université Paul Sabatier. Toulouse, 1992.

CLOSSET J.-L. : D'où proviennent certaines « erreurs » rencontrées chez les élèves et les étudiants en électrocinétique ? Peut-on y remédier ? Bulletin de l'Union des physiciens $n^{\circ} 657$. pp.81/102, 1983.

SALTIEL E. et VIENNOT L. : Questionnaire pour comprendre. Paris : I.R.E.M., 1991.

VIENNOT L. : Common Practice in Elementary Algebra. European Journal of Science Education. Vol.3, N², pp. 183/194, 1981.

VIENNOT L. : Implicit statements in physics : students and constants. Atelier International d'été, Recherche en Didactique de la Physique, La Londe les Maures, pp.355/361, 1983.

\section{RÉSUMÉS}

Nous présentons une étude comparée de deux groupes d'élèves de classes de première, section Electrotechnique, de Lycée Technique. Leurs passés scolaires diffèrent : enseignements classique en seconde pour un groupe et professionnalisé en L. E. P. (Lycée d'Enseignement Professionnel) pour l'autre. Nous avons abordé trois thèmes relatifs à l'électrocinétique à courant continu : les conceptions et leur instabilité, la pratique courante de l'algèbre élémentaire en Physique Appliquée, l'utilisation de relations mathématiques simples. Ces points ont été traités par l'intermédiaire d'un questionnaire papier/crayon. L'analyse des réponses nous a permis de mettre en évidence certaines caractéristiques spécifiques à chaque groupe.

We present a comparative study on two groups of students from first classes in the Electro technical Section of a Technical High School. Both classes having received different school training : a classical second year training for one group and the other professionally trained (in a Professional Technical School - normal level ).We have covered three subjects in relation to Electro kinetics at direct current : the conceptions and their instability, the common practice of elementary algebra, the utilisation of relations elementary mathematics. These points were covered by means of a questionnaire paper/pencil. The analysis of the answers enabled us to put into evidence certain specific characteristics in each group. (Please, note that the classes mentioned in this study are those practiced in the French schooling).

\section{INDEX}

Mots-clés : algèbre, électrocinétique, enseignement, mathématique, raisonnement, relation, technique 


\section{AUTEUR}

\section{B. CALMETTES}

LEMME, Université Paul Sabatier de Toulouse 